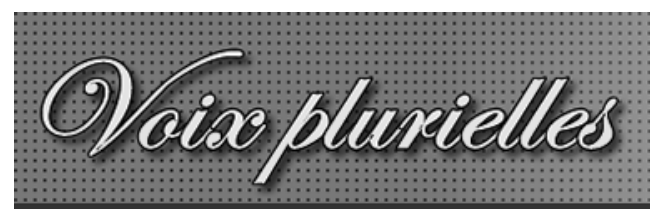

Revue de l'Association des Professeur-e-s de Français des Universités et Collèges Canadiens

\author{
Voix plurielles \\ Volume 4, Numéro 1 : mai 2007
}

Jean B. Ntakirutimana

\title{
Traitement de l'ambiguïté dans les logiciels de traduction automatique en ligne
}

Citation MLA : Ntakirutimana, Jean B. «Titre.» Voix plurielles 4.1 (mai 2007).

(C) Voix plurielles, revue électronique de l'APFUCC 2007. 


\title{
Traitement de l'ambiguïté dans les logiciels de traduction automatique en ligne
}

\author{
Jean B. Ntakirutimana \\ Université Brock
}

Mai 2007

\section{Introduction}

I 1 existe de nos jours plusieurs logiciels de traduction automatique et bon nombre d'entre eux sont accessibles sur l'internet. Ainsi, des moteurs de recherche comme AltaVista ou Yahoo proposent aux internautes d'afficher les pages Web cibles en versions traduites instantanément dans la langue de choix de l'utilisateur. À l'instar d'autres gadgets issus des nouvelles technologies de l'information et de la communication (NTIC), les logiciels de traduction automatique sont largement utilisés partout dans le monde, et certains utilisateurs ne s'interrogent même pas sur l'efficacité réelle de ces nouveaux outils langagiers. L'on comprend alors pourquoi la traduction automatique génère ci et là, et de façon quasi anodine, des perles langagières du genre :

\section{Push the hour hand clockwise:}

- Poussez comme les aiguilles d'une montre l'heure main (1-800-translate)

- Poussez la main d'heure dans le sens des aiguilles d'une montre (Abacho)

2. Bleach When Needed:

Eau de Javel Quand a Eu besoin de (KG Industries Web Site)

\section{Welcome back:}

- Bienvenue en arrière (World Lingo)

- Dos bienvenu (Personal Translator Online)

4. The news got through to them:

- Les nouvelles ont obtenu par à les (Free Text Translator)

5. After a number of injections, my jaw got number:

- Après un certain nombre d'injections, ma mâchoire a reçu le nombre (PROMT-Online) ${ }^{1}$

De telles erreurs font que les connaisseurs des outils langagiers informatisés développent une certaine méfiance envers les logiciels de traduction automatique, pendant que ces derniers continuent à susciter de l'engouement un peu partout dans les régions où les NTIC connaissent une certaine expansion. Eu égard à cette situation, on est en droit de se demander si ces outils de traduction automatique n'auraient pas quelques atouts cachés, ou s'il n'y en aurait pas quelques uns qui seraient réellement efficaces. 
En réponse à ces interrogations, nous avons initié un travail de recherche dont le but était d'évaluer la performance des principaux logiciels de traduction automatique disponibles sur le réseau internet. Le présent papier expose le cheminement qui a été suivi pour évaluer les logiciels-pilotes, objets de cette étude. Ensuite les résultats obtenus sont présentés et analysés comparativement.

Étant donné que les critères d'évaluation des logiciels de traduction en question sont principalement centrés sur leur efficacité dans le traitement des énoncés ambigus, la notion d'ambiguïté sémantique est brièvement présentée au préalable. Par ce bref survol, le lecteur sera amené à comprendre les raisons pour lesquelles une attention particulière a été accordée à la notion d'ambiguïté. La seconde partie décrit le cheminement méthodologique qui a été suivi dans ce travail. La procédure est classique; elle consiste essentiellement à soumettre une même routine à différents outils informatiques, afin de pouvoir évaluer par la suite leur fonctionnement et leur rendement, sur la base de critères uniformes. Il y a lieu de préciser que l'analyse des mécanismes de traitement de l'ambiguïté par les logiciels de traduction automatique nous fournit l'occasion d'analyser en profondeur les processus de fonctionnement des logiciels de traductique, en tenant compte des mécanismes sous-jacents à toute routine de traduction automatique. Cela nous permet ainsi d'établir une typologie sommaire des logiciels de traduction automatique testés. Les résultats de ces analyses sont succinctement présentés dans l'avant-dernière partie de ce document. La dernière partie, quant à elle, propose une évaluation synthétique des outils logiciels ayant fait l'objet de la présente étude, et met en perspective leurs points de convergence respectifs, pour ceux qui en ont, de même que leurs différences.

\section{Ambigü̈té et langues naturelles}

Les langues naturelles sont essentiellement créatives. Cette forme de dynamisme qui leur est inhérente se manifeste sur le plan de la forme et du sens, surtout au niveau des différents mécanismes d'enrichissement lexical et à travers des procédés stylistiques comme la polysémie, l'homonymie et la synonymie, pour ne citer que ceux-là. Généralement, on parle de polysémie lorsqu'un signe linguistique possède des valeurs sémantiques différentes mais néanmoins rapprochées; tandis que l'homonymie caractérise un signe linguistique à valeurs sémantiques différentes et très éloignées les unes des autres. La synonymie, quant à elle, consiste en des signes linguistiques différents mais sémantiquement identiques (Dubois et al. : 2001). Par le biais de ces procédés, des unités lexicales identiques acquièrent des sens différents d'une part et, d'autre part, les concepts ou notions similaires sont exprimés par une grande variété d'énoncés.

Plusieurs penseurs ont élaboré à profusion sur le caractère essentiel de la polysémie, de l'homonymie et de la synonymie dans les langues naturelles (Leibniz, Bréal, Sparck Jones, Balibar-Mrabti, Ullmann, Bally, ...). Soulignons au passage que ces procédés stylistiques participent au dynamisme des langues naturelles en augmentant leurs potentialités expressives et en leur conférant une plus grande vivacité. Néanmoins, par leur diversification des formes et des contenus linguistiques, ces mêmes stratégies stylistiques créent de nombreuses ambiguïtés au sein des langues naturelles.

La haute fréquence de la polysémie, de l'homonymie et de la synonymie en langues naturelles génère, par la même occasion, beaucoup d'ambiguïtés dans le discours. De temps en temps, cette 
ambiguïté constitue un défi en matière de communication, bien que les locuteurs de langues naturelles s'en accommodent assez aisément. Parfois même, les humains se servent délibérément de l'ambiguïté dans le but d'épicer ou de raffiner la langue, tel que l'ont confirmé plusieurs auteurs (Derwing (1973), Ullmann (1973) ou Wittgenstein (1968)). Ce fait est également illustré par les fréquentes conversations à mots couverts entre interlocuteurs qui se connaissent bien, les argots omniprésents dans différents milieux sociolinguistiques, ou par les mots ambigus que l'on est obligé d'interpréter et de réinterpréter dans de nombreuses productions littéraires.

De façon générale, l'ambiguïté en langue naturelle ne semble pas faire obstacle à la communication interhumaine. Les interlocuteurs savent contourner ce léger défi à la communication en développant une panoplie de mécanismes de désambiguïsation : interprétation des sous-entendus, demande de clarifications, détection du sens à travers le contexte, etc. Que ce soit à 1'oral ou à l'écrit, les humains utilisent, souvent de manière instinctive, des règles tacites de désambiguïsation. En règle générale, il est connu que, pour être efficace, la communication doit utiliser des mots suivant une échelle d'acceptions conceptuelles plus ou moins délimitées. L'émetteur et le récepteur doivent se conformer à cette échelle bien qu'une certaine marge soit laissée à l'interprétation individuelle (Schogt (1976), Osgood \& Tzeng (1990), Ullmann (1975)). À l'évidence, tout acte de communication s'accompagne d'une forme de négociation sémantique entre les interlocuteurs. De la même manière qu'un dialogue n'est pas toujours transparent du point de vue sémantique, il est rare qu'un texte à traduire ne contienne des ambiguïtés. Il va sans dire que les traducteurs humains, par leur professionnalisme, savent comment contourner ces ambiguïtés et parviennent même à les éliminer dans la langue cible. Mais qu'en est-il réellement des traducteurs machines?

Sur la base de l'importance et de l'omniprésence de l'ambiguïté dans les langues naturelles, l'idée maîtresse autour de laquelle s'est articulé le présent exercice analytique était de déterminer si les outils de traduction automatiques actuellement disponibles sur le Web sont à même d'appréhender l'ambiguïté sémantique et de la traduire efficacement, à l'instar des traducteurs humains. À cette fin, nous avons observé comment les logiciels de traduction automatique traitent des énoncés ambigus traduits de l'anglais vers le français, selon une procédure qui sera directement abordée ci-dessous.

\section{Méthodologie}

\subsection{Choix des logiciels-pilotes}

Les analyses ont porté sur les logiciels accessibles en ligne et tournant directement sur le réseau internet. Il s'agit de logiciels qui permettent de faire une traduction directe sur le Web sans que l'usager ne soit obligé de les télécharger sur son ordinateur. Le choix de ce genre de logiciels a été dicté par la nécessité d'encrer la présente étude sur des outils réellement existants. De plus, ces derniers semblent être les plus utilisés par les internautes, entre autres en raison de leur forte visibilité sur le Web.

À l'aide du moteur de recherche Google, il a été possible d'accéder aux 17 logiciels de traduction suivants : 
@PROMT translation software ${ }^{2}$

1-800-translate

Abacho

Babel Fish (AltaVista) ${ }^{3}$

Enterprise Translation Server ${ }^{\mathrm{TM}}$ (SDL International)

Free Text Translator (Free Translation)

Google Language Tools (Google)

Im Translator (Smart Link Corporation)

Inter Tran ${ }^{\mathrm{TM}}$

Lost in Translation

Lycos Translation (Lycos)

Personal Translator Online (Linguatec)

PROMT-Online

Reverso (Softissimo)

Systran (Systran)

Translated.net

WorldLingo

En vue d'évaluer les potentialités des logiciels ci-dessus, nous leur avons soumis pour traduction la phrase "FLYING PLANES CAN BE DANGEROUS". Celle-ci fait partie des énoncés classiques utilisés en traductique, lors des tests évaluatifs des systèmes de traduction automatique. Il a été jugé préférable de partir de l'anglais comme langue source afin de faciliter le long processus d'évaluation, tant il est vrai que la plupart des logiciels de traduction automatique en ligne affichent l'anglais comme langue de départ par défaut. De plus, les deux textes ci-dessous ont été composés dans le seul but de permettre l'utilisation de la phrase-test dans des contextes permettant d'en éliminer toute ambiguïté. Comme il est aisé de le comprendre, le premier texte explique en détails pourquoi un avion volant peut être dangereux, tandis que le second explique les dangers reliés à la profession de pilote d'avion.

\section{WHY IS A FLYING PLANE DANGEROUS?}

Because:

It flies at an extremely high speed and can't easily skip obstacles.

Impact at high speed causes much damage.

The fuel used in planes is extremely inflammable.

Therefore, regarding airplanes, specialists usually say: "flying planes can be dangerous".

2. WHY IS THE PROFESSION OF AIR PILOT DANGEROUS?

Because:

A pilot flies a very dangerous craft (plane).

Flying a plane requires high concentration.

One single error can cause the loss of hundreds of lives.

A pilot is the first target for hijackers.

Therefore, regarding the profession of air pilots, specialists usually say: "flying planes can be 
dangerous".

Les deux textes permettent une désambiguïsation contextuelle principalement à trois niveaux :

- Les deux titres numérotés clarifient respectivement le contenu de chaque texte, à savoir « un avion qui vole » (A FLYING PLANE) ainsi que « la profession de pilote d'avion » (THE PROFESSION OF AIR PILOT).

- Les dangers énumérés dans le texte 1 concernent uniquement les avions qui volent, et ceux du texte 2 concernent exclusivement la profession de pilote.

- L'argumentation est organisée de telle sorte que la citation finale soit directement précédée de son contexte d'emploi, afin de lever toute équivoque dans l'énoncé conclusif de chaque texte.

Ainsi, grâce aux trois niveaux de désambiguïsation, nul doute que tout anglophone qui lirait les deux phrases ferait sans peine la part des dangers qui concernent les aéronefs et ceux qui se rapportent à la profession de pilote.

Si tout semble clair pour les lecteurs anglophones humains, qu'en est-il des traducteurs automatiques? La section suivante montre les dix-sept séquences de résultats qui ont été obtenues après avoir successivement soumis les deux textes à chacun des logiciels-pilotes.

\subsection{Traductions obtenues}

\section{Inter $\operatorname{Tran}^{\mathrm{TM}}$}

1. TIENS! C'EST UNE VOLANT L'USINE Dangereux?

parce que

Le mouches à une extrêmement élevé vitesse et can’t facilement sautiller obstacle.

Retentissement à élevé vitesse raisons très tort.

Les essence utilisée dans l'usine c'est extrêmement inflammable.

Par conséquent, en ce qui concerne aéroplanes, spécialiste habituellement dites : 'flying l'usine can être dangereux.

2. TIENS! C'EST LES PROFESSION DE AÉRER PILOTER Dangereux?

parce que

UNE piloter mouches une véritable dangereux ruse ( l'usine ).

Volant une l'usine vouloir élevé concentration.

Une unique mépris can raison les perte de des centaines de vit.

UNE piloter c'est la première point de mire pour pirate de l'air.

Par conséquent, en ce qui concerne les profession de aérer piloter, spécialiste habituellement dites

: "flying l'usine can être dangereux.

1-800-translate

1. POURQUOI EST-CE QU'UN AVION VOLANT EST DANGEREUX?

Parce que:

Il vole à une vitesse extrêmement haute et ne peut pas sauter facilement d'obstacles.

Enfoncez à haute vitesse cause beaucoup endommagez.

Le combustible usagé dans les avions est extrêmement inflammable. 
Par conséquent, les spécialistes disent habituellement concernant avions: les «avions volants peuvent être dangereux.»

\section{POURQUOI EST-CE QUE LA PROFESSION D'AIR EST PILOTE DANGEROUS?}

Parce que:

Un pilote pilote une habileté très dangereuse (avion).

Piloter un avion exige la haute concentration.

Une erreur seule peut causer la perte de centaines de vies.

Un pilote est la première cible pour les pirates de l'air.

Par conséquent, les spécialistes disent habituellement concernant la profession de pilotes de l'air: les «avions volants peuvent être dangereux.»

Personal Translator Online (Linguatec )

\section{Un AVION VOLANT, POURQUOI EST-IL DANGEREUX?}

Parce que:

Il vole à un extrêmement la vitesse grande et la boîte que t de ' facilement saute les obstacles.

L'impact à la vitesse grande cause dégâts.

Le carburant utilisé dans les avions est extrêmement inflammable.

Par conséquent, en regardant les avions, les spécialistes habituellement disons: Les avions volants de "peuvent être" dangereux.

2. L'AFFIRMATION DU PILOTE AERIEN, POURQUOI EST-ELLE DANGEREUSE?

Parce que:

Un pilote pilote un métier (avion) très dangereux.

Le pilotage d'un avion demande la concentration haute.

Une seule erreur peut causer la perte des centaines des vies.

Un pilote est la première cible pour les pirates.

Par conséquent regarder l'affirmation de l'air pilote, les spécialistes disent habituellement: Les avions volants de "peuvent être" dangereux.

\section{WorldLingo}

\section{POURQUOI UN AVION DE VOL EST-IL DANGEREUX?}

Puisque :

Il vole extrêmement à un à grande vitesse et ne peut pas facilement sauter des obstacles.

L'impact à la vitesse endommage beaucoup.

Le carburant utilisé dans des avions est extrêmement inflammable.

Par conséquent, concernant des avions, de spécialistes parole habituellement : les « avions de vol peuvent être dangereux $\gg$.

\section{POURQUOI LA PROFESSION DU PILOTE D'AIR EST-ELLE DANGEREUSE?}

Puisque :

Un pilote vole un métier très dangereux (avion).

Voler un avion exige la concentration élevée.

Une erreur simple peut causer la perte de centaines de vies.

Un pilote est la première cible pour des pirates de l'air.

Par conséquent, concernant la profession des pilotes d'air, de spécialistes parole habituellement : les « avions de vol peuvent être dangereux ». 


\section{Translated.net}

\section{POURQUOI UN AVION DE VOL EST-IL DANGEREUX?}

Puisque :

Il vole extrêmement à un à grande vitesse et ne peut pas facilement sauter des obstacles.

L'impact à la vitesse endommage beaucoup.

Le carburant utilisé dans des avions est extrêmement inflammable.

Par conséquent, concernant des avions, de spécialistes parole habituellement : les « avions de vol peuvent être dangereux $\gg$.

\section{POURQUOI LA PROFESSION DU PILOTE D'AIR EST-ELLE DANGEREUSE ?}

Puisque :

Un pilote vole un métier très dangereux (avion).

Voler un avion exige la concentration élevée.

Une erreur simple peut causer la perte de centaines de vies.

Un pilote est la première cible pour des pirates de l'air.

Par conséquent, concernant la profession des pilotes d'air, de spécialistes parole habituellement : les « avions de vol peuvent être dangereux».

Free Text Translator (Free Translation)

\section{POURQUOI UN AVION VOLANT EST DANGEREUX?}

Parce que :

Il vole à un extrêmement rapide et ne peut pas sauter facilement des obstacles.

Influer à toute vitesse cause de beaucoup de dommages.

Le carburant utilisé dans les avions est extrêmement inflammable.

Donc, en ce qui concerne les avions, les spécialistes disent d'habitude : « Voler les avions peuvent être dangereux $\gg$.

\section{POURQUOI LA PROFESSION DE PILOTE D’AIR EST-il DANGEREUSE ?}

Parce que :

Un pilote vole un métier très dangereux (l'avion).

Voler un avion exige l'haute concentration.

Une erreur seule peut causer la perte de centaines de vies.

Un pilote est le premier cible pour les terroristes.

Donc, en ce qui concerne la profession de pilotes d'air, les spécialistes disent d'habitude : «Voler les avions peuvent être dangereux $»$.

\section{Enterprise Translation Server ${ }^{\mathrm{TM}}$ (SDL International)}

\section{POURQUOI UN AVION VOLANT EST DANGEREUX ?}

Parce que :

Il vole à un extrêmement rapide et ne peut pas sauter facilement des obstacles.

Influer à toute vitesse cause de beaucoup de dommages.

Le carburant utilisé dans les avions est extrêmement inflammable.

Donc, en ce qui concerne les avions, les spécialistes disent d'habitude : «Voler les avions peuvent être dangereux $»$.

2. POURQUOI LA PROFESSION DE PILOTE D’AIR EST-il DANGEREUSE? 
Parce que :

Un pilote vole un métier très dangereux (l'avion).

Voler un avion exige l'haute concentration.

Une erreur seule peut causer la perte de centaines de vies.

Un pilote est le premier cible pour les terroristes.

Donc, en ce qui concerne la profession de pilotes d'air, les spécialistes disent d'habitude : « Voler les avions peuvent être dangereux ».

\section{Abacho}

\section{POURQUOI UN AVION VOLANT EST DANGEREUX ?}

Puisque :

Il vole à une extrêmement grande vitesse et ne peut pas facilement sauter des obstacles.

L'impact à la grande vitesse provoque beaucoup de dommage.

Le combustible utilisé dans les avions est extrêmement inflammable.

Donc, concernant les aéroplanes, les spécialistes disent d'habitude: "les avions volant peuvent être dangereux".

\section{POURQUOI LA PROFESSION D'AIR EST PILOTE DANGEREUX ?}

Puisque :

un pilote vole un artisanat très dangereux (l'avion).

En volant un avion exige la haute concentration.

Une erreur simple peut provoquer la perte de centaines de vies.

Un pilote est la première cible pour les pirates de l'air.

Donc, concernant la profession de pilotes aériens, les spécialistes disent d'habitude: "les avions volant peuvent être dangereux".

@.PROMT Translation Software (PROMT)

\section{POURQUOI UN AVION VOLANT EST DANGEREUX ?}

Puisque :

Il vole à une extrêmement grande vitesse et ne peut pas facilement sauter des obstacles.

L'impact à la grande vitesse provoque beaucoup de dommage.

Le combustible utilisé dans les avions est extrêmement inflammable.

Donc, concernant les aéroplanes, les spécialistes disent d'habitude: "les avions volant peuvent être dangereux".

2. POURQUOI LA PROFESSION D’AIR EST PILOTE DANGEREUX ?

Puisque :

un pilote vole un artisanat très dangereux (l'avion).

En volant un avion exige la haute concentration.

Une erreur simple peut provoquer la perte de centaines de de vies.

Un pilote est la première cible pour les pirates de l'air.

Donc, concernant la profession de pilotes aériens, les spécialistes disent d'habitude: "les avions volant peuvent être dangereux".

\section{Im Translator (Smart Link Corporation)}




\section{POURQUOI UN AVION VOLANT EST DANGEREUX ?}

Parce que :

Il vole à une extrêmement grande vitesse et ne peut pas facilement sauter des obstacles.

L'impact à la grande vitesse provoque beaucoup de dommage.

Le combustible utilisé dans les avions est extrêmement inflammable.

Donc, quant aux aéroplanes, les spécialistes disent d'habitude: "les avions volant peuvent être dangereux".

2. POURQUOI LA PROFESSION D’AIR EST PILOTE DANGEREUX ?

Parce que :

Un pilote vole un artisanat très dangereux (l'avion).

En volant un avion exige la haute concentration.

Une erreur simple peut provoquer la perte de centaines de vies.

Un pilote est la première cible pour les pirates de l'air.

Donc, quant à la profession de pilotes aériens, les spécialistes disent d'habitude: "les avions volant peuvent être dangereux".

\section{PROMT-Online}

\section{POURQUOI UN AVION VOLANT EST DANGEREUX?}

Parce que :

Il vole à une extrêmement grande vitesse et ne peut pas facilement sauter des obstacles.

L'impact à la grande vitesse provoque beaucoup de dommage.

Le combustible utilisé dans les avions est extrêmement inflammable.

Donc, quant aux aéroplanes, les spécialistes disent d'habitude: "les avions volant peuvent être dangereux".

2. POURQUOI LA PROFESSION D’AIR EST PILOTE DANGEREUX ?

Parce que :

Un pilote vole un artisanat très dangereux (l'avion).

En volant un avion exige la haute concentration.

Une erreur simple peut provoquer la perte de centaines de vies.

Un pilote est la première cible pour les pirates de l'air.

Donc, quant à la profession de pilotes aériens, les spécialistes disent d'habitude: "les avions volant peuvent être dangereux".

\section{Reverso (Softissimo)}

\section{POURQUOI UN AVION VOLANT EST DANGEREUX ?}

Parce que :

Il vole à extrêmement à grande vitesse et ne peut pas facilement sauter des obstacles.

L'impact cause à grande vitesse beaucoup de dégâts.

Le carburant utilisé dans des avions est extrêmement inflammable.

Donc, quant aux avions, les spécialistes disent d'habitude : “des avions volants peuvent être dangereux".

2. POURQUOI EST LA PROFESSION DE PILOTE AÉRIEN DANGEREUX ? 
Parce que :

Un pilote transporte par avion un métier très dangereux (l'avion).

Le vol d'un avion exige la haute concentration.

Une erreur seule(simple) peut causer la perte de centaines de vies.

Un pilote est la première cible pour des pirates de l'air.

Donc, quant à la profession de pilotes aériens, les spécialistes disent d'habitude : “des avions volants peuvent être dangereux".

\section{Lycos Translation (Lycos) $)^{4}$}

\section{POURQUOI UN AVION DE VOL EST-IL DANGEREUX?}

Puisque : Il vole extr $\tilde{A}^{\mathrm{a}}$ mement $\tilde{\mathrm{A}}$ un $\tilde{\mathrm{A}}$ grande vitesse et ne peut pas facilement sauter des obstacles.

L'impact Ã la vitesse endommage beaucoup.

Le carburant utilis $\tilde{A} \subset$ dans des avions est extr $\tilde{A}^{\mathrm{a}}$ mement inflammable.

Par cons $\tilde{A} @$ quent, concernant des avions, les sp $\tilde{A} \subset$ cialistes disent habituellement : les $\hat{A} \ll \hat{A}$ avions de vol peuvent $\tilde{A}^{a}$ tre dangereux $\hat{A} \hat{A} »$.

2. POURQUOI LA PROFESSION DU PILOTE D’AIR EST-ELLE DANGEREUSE?

Puisque :

Un pilote vole un $m \tilde{A} @$ Ctier $\operatorname{tr} \tilde{A}$ ”s dangereux (avion).

Voler un avion exige la concentration $\tilde{A} @$ Clev $\tilde{A} \subset$ Ce.

Une erreur simple peut causer la perte de centaines de vies.

Un pilote est la premi Ã re cible pour des pirates de l'air.

Par cons $\tilde{A} \subseteq$ quent, concernant la profession des pilotes d'air, les sp $\tilde{A} \Subset$ cialistes disent habituellement : les $\hat{A}$ «Â avions de vol peuvent $\tilde{A}^{a}$ tre dangereux $\hat{A} \hat{A} »$.

\section{Babel Fish (AltaVista)}

\section{POURQUOI Un AVION De VOL EST-IL DANGEREUX?}

Puisque :

Il vole extrêmement à un à grande vitesse et ne peut pas facilement sauter des obstacles.

L'impact à la grande vitesse endommage beaucoup.

Le carburant utilisé dans des avions est extrêmement inflammable.

Par conséquent, concernant des avions, les spécialistes disent habituellement : les «avions de vol peuvent être dangereux»».

2. POURQUOI LA PROFESSION DU PILOTE D'AIR EST-ELLE DANGEREUSE?

Puisque :

Un pilote vole un métier très dangereux (avion).

Voler un avion exige la concentration élevée.

Une erreur simple peut causer la perte de centaines de vies.

Un pilote est la première cible pour des pirates de l'air.

Par conséquent, concernant la profession des pilotes d'air, les spécialistes disent habituellement :

les «avions de vol peuvent être dangereux». 


\section{Systran (Systran)}

\section{POURQUOI UN AVION DE VOL EST-IL DANGEREUX?}

Puisque :

Il vole extrêmement à un à grande vitesse et ne peut pas facilement sauter des obstacles.

L'impact à la vitesse endommage beaucoup.

Le carburant utilisé dans des avions est extrêmement inflammable.

Par conséquent, concernant des avions, les spécialistes disent habituellement : les « avions de vol peuvent être dangereux $\gg$.

2. POURQUOI LA PROFESSION DU PILOTE D'AIR EST-ELLE DANGEREUSE?

Puisque :

Un pilote vole un métier très dangereux (avion).

Voler un avion exige la concentration élevée.

Une erreur simple peut causer la perte de centaines de vies.

Un pilote est la première cible pour des pirates de l'air.

Par conséquent, concernant la profession des pilotes d'air, les spécialistes disent habituellement : les « avions de vol peuvent être dangereux».

\section{Google Language Tools (Google)}

\section{POURQUOI UN AVION DE VOL EST-IL DANGEREUX?}

Puisque :

Il vole extrêmement à un à grande vitesse et ne peut pas facilement sauter des obstacles.

L'impact à la vitesse endommage beaucoup.

Le carburant utilisé dans des avions est extrêmement inflammable.

Par conséquent, concernant des avions, les spécialistes disent habituellement : les « avions de vol peuvent être dangereux $\gg$.

\section{POURQUOI LA PROFESSION DU PILOTE D’AIR EST-ELLE DANGEREUSE?}

Puisque :

Un pilote vole un métier très dangereux (avion).

Voler un avion exige la concentration élevée.

Une erreur simple peut causer la perte de centaines de vies.

Un pilote est la première cible pour des pirates de l'air.

Par conséquent, concernant la profession des pilotes d'air, les spécialistes disent habituellement : les « avions de vol peuvent être dangereux».

\section{Lost in Translation}

\section{POURQUOI Un AVION de VOL EST-IL DANGEREUX?}

Puisque :

Il vole extrêmement à un à grande vitesse et le can_t sautent facilement des obstacles.

L'impact à la grande vitesse endommage beaucoup.

Le carburant utilisé dans des avions est extrêmement inflammable.

Par conséquent, concernant des avions, les spécialistes disent habituellement :_les avions de vol 
peuvent être dangereux .

2. POURQUOI LA PROFESSION DU PILOTE D’AIR EST-ELLE DANGEREUSE?

Puisque :

Un pilote vole un métier très dangereux (avion).

Voler un avion exige la concentration élevée.

Une erreur simple peut causer la perte de centaines de vies.

Un pilote est la première cible pour des pirates de l'air.

Par conséquent, concernant la profession des pilotes d'air, les spécialistes disent habituellement :

_ les avions de vol peuvent être dangereux _.

\section{Analyse des résultats}

Les résultats obtenus semblent suffisamment éloquents en eux-mêmes. Aucun logiciel de traduction automatique n'a été capable de désambiguïser la phrase "FLYING PLANES CAN BE $D A N G E R O U S$ ", malgré tous les artifices qui avaient été déployés dans le but de rendre transparents ses deux contextes d'emploi.

\subsection{Structure générale des logiciels de traduction automatique}

En analysant les différentes traductions générées par les logiciels-tests, avec une insistance particulière sur la dernière phrase, il est probable que d'aucuns soient amenés à réfléchir sur le mode de fonctionnement des logiciels en question. Notons qu'il est communément accepté que, grosso modo, la structure d'un logiciel de traduction se ramène à deux composantes essentielles, à savoir un moteur lexical et un moteur syntaxique.

Le moteur lexical est une forme de dictionnaire multilingue. Il est constitué de listes lexicales dans chacune des langues prises en charge par le logiciel. Chaque unité lexicale dans une langue donnée est reliée à ses équivalents sémantiques dans les autres langues. Plus les listes lexicales seront riches, plus le moteur lexical établira des équivalents sémantiques plus pointus, déterminés suivant des calculs lexicostatistiques assez complexes, et que les contraintes d'ordre spatial ne nous permettent pas d'expliquer en détail dans le cadre de cette étude.

Le moteur syntaxique, quant à lui, sert à la reconnaissance des phrases. Il fonctionne comme un analyseur grammatical, décomposant chaque phrase de la langue de départ en ses structures syntaxiques. Cela permet ainsi d'établir des structures syntaxiques équivalentes dans la langue d'arrivée. À titre illustratif, c'est grâce au moteur syntaxique qu'une structure interrogative avec «why» en anglais sera transformée en « pourquoi est-ce que », ou en une phrase interrogative à inversion VerbeSujet, le tout précédé de « pourquoi ... ».

Les moteurs syntaxiques varient d'un logiciel à l'autre. Certains moteurs de faible envergure se contentent de transposer telles quelles les structures syntaxiques de la langue source dans la langue cible, traduisant ainsi les phrases mot à mot. D'autres moteurs de plus grande portée sont capables d'analyser des phrases relativement longues. Ce sont des moteurs pareils qui permettent d'établir dans la langue cible des phrases à structures syntaxiques relativement acceptables.

Étant donné que l'ambiguïté des phrases comme celles analysées dans cette étude ne peut être levée que grâce à la compréhension du contexte d'emploi, il va sans dire que c'est surtout la 
portée du moteur syntaxique qui détermine le degré d'acuité et de désambiguïsation du logiciel. L'ensemble sera évidemment enrichi et clarifié à l'aide du vocabulaire fourni par le moteur lexical. Le résultat est que la valeur sémantique de toute phrase traduite automatiquement est le produit du travail accompli par les deux principaux moteurs de la machine traductionnelle, à savoir le moteur syntaxique et le moteur lexical.

Tenant compte des structures ci-dessus, une analyse typologique des logiciels de traduction automatique qui ont été testés s'est imposée comme étant le meilleur moyen pour rendre compte du mode de fonctionnement des outils en question.

\subsection{Typologie des logiciels-tests}

Au cours de l'analyse typologique des logiciels-tests, chacun d'eux a été évalué au niveau $\mathrm{du}$ fonctionnement du moteur lexical, de la puissance et de la portée du moteur syntaxique, ainsi qu'au niveau de la qualité linguistique des traductions fournies. Les détails inhérents à ce travail de typologie ne sont pas indispensables dans le cadre de cet article. Le plus important à retenir est que les logiciels dont les résultats sont relativement proches ont été classés à proximité des uns aux autres $^{5}$. En outre, les logiciels groupés et analysés ensemble sont ceux qui fonctionnent de manière identique; autrement dit, ceux qui ont traduit les deux textes de façon parfaitement identique. Le résultat final de ce travail typologique pourrait être synthétisé ainsi :

Inter Tran ${ }^{\mathrm{TM}}$

moteur lexical extrêmement faible

moteur syntaxique extrêmement faible

portée du moteur syntaxique : entre 2 blancs typographiques

traduction presque mot à mot

trop d'erreurs lexicales et syntaxiques

quelques unités lexicales non traduites

1-800-translate

moteur lexical extrêmement faible

moteur syntaxique extrêmement faible

portée du moteur syntaxique : entre 2 blancs typographiques

traduction presque mot à mot

trop d'erreurs lexicales et syntaxiques

Personal Translator Online (Linguatec )

moteur lexical extrêmement faible

moteur syntaxique extrêmement faible

portée du moteur syntaxique : entre 2 blancs typographiques

traduction presque mot à mot

trop d'erreurs lexicales et syntaxiques

WorldLingo

Translated.net

moteur lexical très faible

moteur syntaxique très faible

portée du moteur syntaxique : entre 2 signes de ponctuation 
traduction de très courts énoncés

beaucoup d'erreurs lexicales et syntaxiques

Free Text Translator (Free Translation)

Enterprise Translation Server ${ }^{\mathrm{TM}}$ (SDL International)

moteur lexical très faible

moteur syntaxique très faible

portée du moteur syntaxique : entre 2 signes de ponctuation

traduction de très courts énoncés

beaucoup d'erreurs lexicales et syntaxiques

Abacho

@PROMT Translation Software (PROMT)

moteur lexical faible

moteur syntaxique faible

portée du moteur syntaxique : entre 2 signes de ponctuation

traduction de courts énoncés

quelques erreurs lexicales et syntaxiques

Im Translator (Smart Link Corporation)

PROMT-Online

moteur lexical faible

moteur syntaxique faible

portée du moteur syntaxique : entre 2 signes de ponctuation

traduction de courts énoncés

quelques erreurs lexicales et syntaxiques

Reverso (Softissimo)

moteur lexical faible

moteur syntaxique faible

portée du moteur syntaxique : entre 2 signes de ponctuation traduction de courts énoncés

quelques erreurs lexicales et syntaxiques

Lycos Translation (Lycos)

moteur lexical faible

moteur syntaxique faible

portée du moteur syntaxique : entre 2 signes de ponctuation

traduction de courts énoncés

quelques erreurs lexicales et syntaxiques

Babel Fish (AltaVista)

moteur lexical faible

moteur syntaxique faible

portée du moteur syntaxique : entre 2 signes de ponctuation traduction de courts énoncés

quelques erreurs lexicales et syntaxiques

Systran (Systran) 
Google Language Tools (Google)

moteur lexical faible

moteur syntaxique faible

portée du moteur syntaxique : entre 2 signes de ponctuation

traduction de courts énoncés

quelques erreurs lexicales et syntaxiques

Lost in Translation

moteur lexical très faible et partiellement fonctionnel

moteur syntaxique très faible

portée du moteur syntaxique : entre 2 signes de ponctuation

traduction de très courts énoncés

beaucoup d'erreurs lexicales et syntaxiques

Malgré l'abondance des sites Web qui mettent des logiciels de traduction automatique à la disposition des internautes, un observateur averti pourra aisément se rendre compte que ce sont souvent les mêmes logiciels qui sont utilisés dans les différents sites Web, avec prédominance des produits de la compagnie Systran et PROMT. Ces derniers se partagent équitablement un marché de 10 sites Web sur un ensemble de 17, soit 5 sites Web chacun. Les produits des compagnies SDL et WorldLingo sont respectivement présents dans seulement 2 sites, alors que les 3 logiciels restants ne sont représentés qu'une seule fois chacun. Nous reviendrons succinctement sur ce constat; mais il importe de souligner, dès à présent, qu'aucun des logiciels testés n'a été à la hauteur de sa tâche.

\section{Synthèse}

Après évaluation des dix-sept logiciels-pilotes, force est de reconnaître qu'aucun d'eux n'a été capable de fournir une traduction pleinement satisfaisante, principalement à cause de nombreuses carences aux niveaux lexical et syntaxique. De plus, tous les logiciels se sont avérés inefficaces face à l'ambiguïté. Aucun logiciel n'a pu rendre compte de la différence, pourtant essentielle, entre la phrase conclusive du premier texte et la phrase conclusive du second texte. Par conséquent, les logiciels testés ont tous échoué l'épreuve de la désambiguïsation.

\subsection{Carences lexico-syntaxiques}

Un des exemples de l'incompétence lexicale de base est que, dans le texte 2 concernant la profession de pilote, beaucoup de logiciels testés n'ont pas pu traduire convenablement “flies» du verbe «to fly» (piloter), «craft» (appareil / engin), ainsi que plusieurs autres mots assez simples. Il y a même lieu de relever des cas extrêmes comme celui de Inter Tran ${ }^{\mathrm{TM}}$ qui s'est révélé incapable de traduire «can», ou le cas de World Lingo qui a confondu un verbe (say) avec un substantif (parole). Dans ce dernier cas, le contexte syntaxique et, plus particulièrement, la présence d'un adverbe (usually) aurait laissé présager un verbe plutôt qu'un nom, surtout que ce principe de relations privilégiées entre un adverbe et un verbe demeure fondamental aussi bien en anglais qu'en français.

À un niveau lexico-syntaxique légèrement plus complexe, les logiciels examinés ont tous accusé des carences de taille qui les ont empêchés de reconnaître et de traduire correctement des 
expressions du genre «Impact at high speed». Une autre incompétence lexico-syntaxique notoire s'est manifestée dans la non reconnaissance de l'expression collocationnelle «flying a plane». Curieusement, les seuls logiciels qui ont convenablement traduit l'expression en question sont 1-800-translate et Personal Translator Online. Malheureusement, ces derniers ont fait preuve d'handicaps lexico-syntaxiques plus sérieux dans d'autres phrases.

\section{2. Échec dans la désambiguïsation}

D'aucuns s'accordent pour dire qu'un mot isolé n'a généralement pas de valeur sémantique fixe. Son sens demeure ambigu tant qu'il n'a pas été complété par celui des unités lexicales voisines; le degré de voisinage entre les mots étant, quant à lui, déterminé par les structures syntaxiques de la langue. Ainsi, un logiciel qui traduit mot à mot ne peut convenablement rendre compte du sens d'un énoncé, car chaque mot y est considéré comme une entité autonome. Le résultat est souvent un énoncé incohérent où bon nombre de mots sont inadaptés au contexte ou sont imprécis, donc ambigus. C'est ainsi que des logiciels comme Inter Tran ${ }^{\mathrm{TM}}$, 1800 translate ou Personal Translator Online ont complètement failli au test de la désambiguïsation, car la portée maximale de leurs moteurs syntaxiques se limite au blanc typographique.

Tel que présenté antérieurement au point 3.2, les autres logiciels du groupe ont des moteurs syntaxiques relativement efficaces. Cependant, la portée maximale de ces derniers se situe entre deux signes de ponctuation, peu importe leur nature. C'est dans cette situation que le double-point ( : ) est susceptible d'arrêter le moteur syntaxique. Dès lors, il devient évident que la dernière phrase "flying planes can be dangerous" ne peut être désambiguïsée, étant donné que son sens est déterminé par l'énoncé précédant les deux points. En conséquence, tous les logiciels, même les plus performants, ont échoué la traduction de la phrase-piège, car aucun d'eux n'a pu faire le lien entre la profession de pilote (air pilot) et le fait de piloter un avion (flying plane), d'où un traitement inadéquat de l'ambiguïté. Il va sans dire que ce fait constitue une preuve supplémentaire des limites de la traduction automatique.

\subsection{Au-delà de la traduction automatique}

À partir de tests de logiciels similaires à ceux décrits dans la présente étude, beaucoup de spécialistes de la traductique ont conclu que, à l'état actuel des connaissances et du Traitement Automatique des Langues Naturelles (TALN), il n'est pas réaliste de parler d'une traduction automatique de la langue naturelle, mais plutôt d'une Aide à la Traduction (AT) que d'autres qualifient de Traduction Assistée par Ordinateur (TAO).

Cette nouvelle approche remplace le moteur syntaxique par un moteur d'alignement. Celui-ci met en parallèle des phrases d'une langue source et leurs équivalents en langue cible, tels qu'établis par des traducteurs experts humains. L'ensemble des phrases disponibles pour alignement constitue la mémoire de traduction; une mémoire qui s'enrichit chaque fois que de nouveaux textes sont traduits. En effet, à chaque nouvelle requête de traduction, le système d'aide à la traduction détecte des phrases similaires à celles déjà traduites et alignées. Par la suite, il propose des équivalences, phrase par phrase. L'étape subséquente revient au traducteur qui doit valider, accepter après modifications, ou rejeter chaque phrase traduite par la machine. Bien entendu, le système dispose d'un dictionnaire qui tourne en arrière-fond et qui complète le travail du moteur d'alignement en 
suggérant des traductions de certains mots nouveaux. C'est un concept innové de dictionnaire dynamique, graduellement mis à jour au fur et à mesure que la mémoire de traduction s'enrichit de nouvelles phrases.

Malgré ses performances, cette nouvelle approche demeure encore à l'étape expérimentale et n'est pas très vulgarisée comme l'est la traduction automatique. En effet, des tribunes vantant des logiciels de traduction automatique comme ceux de Systran, de Softissimo, etc. sont légions sur le Web. De plus, beaucoup d'usagers, surtout les apprenants de langues secondes, parfois même des responsables d'entreprises, continuent à croire en l'efficacité des outils de traduction automatique et les utilisent en toute confiance.

$\mathrm{Au}$ cœur de cette prolifération de logiciels de traduction automatique se démarque un fait anodin. À quelques exceptions près, tous les sites Web de traduction automatique gratuite proposent des liens vers des pages qui font la publicité de services linguistiques tels que l'apprentissage des langues secondes, l'aide à la rédaction, l'archivage numérisée de documents, la traduction humaine payante, etc. Certains sites font également la promotion d'outils langagiers comme des logiciels parfois très performants des appareils électroniques, des manuels d'apprentissage de langues, des dictionnaires ou d'autres genres de livres. Une raison vraisemblable derrière la prolifération de logiciels de traduction sur la toile serait que les compagnies qui les disséminent sur le Web veulent s'en servir comme outils de publicité. En d'autres termes, c'est une forme déguisée de marketing, destinée à appâter une clientèle peu avertie afin de l'amener à consommer des biens et services langagiers qui, cette fois-ci, se vendent à prix d'or.

\section{Conclusion}

Les outils de traductique disponibles sur le Web sont inaptes à fournir des traductions satisfaisantes. Certains ne semblent même pas se préoccuper de la qualité en matière de traduction, tel qu'illustré par les exemples de traductions analysés dans ce document. C'est ainsi que Lost in Translation tourne au ridicule le principe même de la traduction automatique, affirmant sans ambages que les logiciels de traduction automatique ne sont pas à prendre au sérieux, du fait qu'il dénaturent la structure du texte-source : «Enter English Text and 'Babelize'it» (Tashian. 2007-03-14 http://www.tashian.com/multibabel/P). Dans tous les cas, il a été maintes fois prouvé que l'objectif d'une traduction entièrement automatisée est encore loin d'être atteint, quelles que soient les langues naturelles de départ ou d'arrivée. Il n'est donc pas trop exagéré d'affirmer que la plupart des sites de traduction automatique constituent surtout des tribunes pour la promotion de produits des grandes compagnies comme Systran, PROMT, Softissimo et consorts. Un appel à la prudence est donc lancé à l'endroit des utilisateurs novices qui prennent à la lettre ou au mot, pour être plus précis les résultats de leurs logiciels de «Traduction Automatique ». Tant il est vrai que les vérifications qui ont été faites au sujet de l'anglais et du français restent valables pour toute autre langue naturelle.

\section{Références}

\section{Ouvrages :}


Balibar-Mrabti, A. "Synonymie abstraite et synonymie concrète en syntaxe ». Langages 128 (1997) : 2550.

Bally, C. Traité de stylistique française. 2 vols. Genève : Goerg \& Cie S. A., 1963.

Derwing, B. L. Transformational Grammar as a Theory of Language Acquisition. A Study in the Empirical, Conceptual and Methodological Foundations of Contemporary Linguistics. New York : Cambridge University Press, 1973.

Dubois, J et al. Dictionnaire de linguistique. Paris : Larousse, 2001.

Osgood, C. E. \& O. C. S.Tzeng, Language, Meaning and Culture. The Selected Papers of C. E. Osgood. New York : Praeger, 1990.

Schogt, G. H. Sémantique synchronique : Synonymie, homonymie, polysémie. Toronto : University of Toronto Press, 1976.

Sparck Jones, K. Synonymy and Semantic Classification. Edinburgh : Edinburgh University Press, 1986.

Ullmann, S. Meaning and Style. Collected Papers. New York : Harper \& Row, 1973.

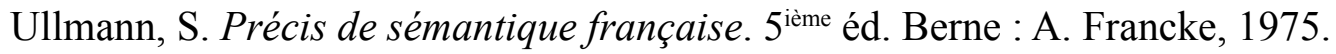

Wittgenstein, L. Philosophical Investigations. The English text of the third edition translated by G. E. M. Anscombe. New York : Macmillan Publishing Co. Inc., 1968.

\section{Sites Web :}

@PROMT translation software. 2007-03-14 http://www.e-promt.com/en/

1-800-translate. 2007-03-14 http://www.1-800-translate.com/machine\%5Ftrans/

Abacho. 2007-03-14 <http://www.fr.abacho.com/uebersetzer.html >

Babel Fish (AltaVista). 2007-03-14 http://babelfish.altavista.com/tr $>$

Enterprise Translation ServerTM (SDL International). 2007-03-14 〈http://www.sdlintl.com/

Free Text Translator (Free Translation). 2007-03-14 http://www.freetranslation.comp

Google Language Tools (Google). 2007-03-14 http://www.google.fr/language tools?hl=en

Im Translator (Smart Link Corporation). 2007-03-14 $\Varangle$ http://translation.imtranslator.net $>$

Inter Tran ${ }^{\text {TM }}$. 2007-03-14 $\langle$ http://www.tranexp.com $>$

Lost in Translation. 2007-03-14 4 http://www.tashian.com/multibabel/

Lycos Translation (Lycos). 2007-03-14 〈http://www.lycos.co.uk/travel/systran.html

Personal Translator Online (Linguatec). 2007-03-14 http://www.linguatec.net/onlineservices $\mathrm{ptp}$

PROMT-Online. 2007-03-14 http://translation2.paralink.com/

Reverso (Softissimo). 2007-03-14<http://www.softissimo.com/>

Systran (Systran). 2007-03-14<http:/www.systransoft.com/>

Translated.net. 2007-03-14 http://free.translated.net/

WorldLingo. 2007-03-14 http://www.worldlingo.com/wl/Translate

\section{Notes}

${ }^{1}$ Ce genre de perles langagières sont surtout très fréquentes dans les modes d'emploi de produits commerciaux. Elles sont aussi de plus en plus présentes dans les travaux scolaires, plus particulièrement chez les apprenants de langues 
secondes.

${ }^{2}$ Les adresses URL des sites hébergeant ces logiciels sont disponibles dans la section des références.

${ }^{3}$ Le nom entre parenthèses indique la compagnie propriétaire du site Web si celle-ci s'est bien identifiée sur le site Web.

${ }^{4}$ L'interface de traduction de Lycos utilisée ici a du mal à afficher les lettres accentuées de la langue française. Il est facile de deviner que la page en question a été initialement conçue pour afficher des textes en langue anglaise, à l'instar de l'ensemble du site Web qui est en anglais.

${ }^{5}$ Ce sont souvent des sites Web qui tournent à partir d'une version plus ou moins modifiée d'un même traducticiel souche. 